\title{
The Viscosity Approximation Forward-Backward Splitting Method for Zeros of the Sum of Monotone Operators
}

\author{
Oganeditse Aaron Boikanyo \\ Department of Mathematics and Statistical Sciences, Botswana International University of Science and Technology, \\ Private Bag Box 16, Palapye, Botswana
}

Correspondence should be addressed to Oganeditse Aaron Boikanyo; boikanyoa@gmail.com

Received 8 September 2015; Accepted 8 December 2015

Academic Editor: Sergei V. Pereverzyev

Copyright (C) 2016 Oganeditse Aaron Boikanyo. This is an open access article distributed under the Creative Commons Attribution License, which permits unrestricted use, distribution, and reproduction in any medium, provided the original work is properly cited.

\begin{abstract}
We investigate the convergence analysis of the following general inexact algorithm for approximating a zero of the sum of a cocoercive operator $A$ and maximal monotone operators $B$ with $D(B) \subset H: x_{n+1}=\alpha_{n} f\left(x_{n}\right)+\gamma_{n} x_{n}+\delta_{n}\left(I+r_{n} B\right)^{-1}\left(I-r_{n} A\right) x_{n}+e_{n}$, for $n=1,2, \ldots$, for given $x_{1}$ in a real Hilbert space $H$, where $\left(\alpha_{n}\right),\left(\gamma_{n}\right)$, and $\left(\delta_{n}\right)$ are sequences in $(0,1)$ with $\alpha_{n}+\gamma_{n}+\delta_{n}=1$ for all $n \geq 1,\left(e_{n}\right)$ denotes the error sequence, and $f: H \rightarrow H$ is a contraction. The algorithm is known to converge under the following assumptions on $\delta_{n}$ and $e_{n}$ : (i) $\left(\delta_{n}\right)$ is bounded below away from 0 and above away from 1 and (ii) $\left(e_{n}\right)$ is summable in norm. In this paper, we show that these conditions can further be relaxed to, respectively, the following: (i) $\left(\delta_{n}\right)$ is bounded below away from 0 and above away from $3 / 2$ and (ii) $\left(e_{n}\right)$ is square summable in norm; and we still obtain strong convergence results.
\end{abstract}

\section{Introduction}

Let $H$ be a real Hilbert space endowed with the inner product $\langle\cdot, \cdot\rangle$ and norm $\|\cdot\|$. Let us consider the problem

find a point $x \in H$ such that $0 \in A x+B x$,

where $A$ and $B$ are maximal monotone operators. The literature on problem (1) exists (see [1-5] and the references therein). Note that two possibilities exist here: either $A+B$ is maximal monotone or $A+B$ is not maximal monotone. (For conditions that ensure that $A+B$ is maximal monotone, we refer the reader to [6].) One of the iterative procedures used to solve problem (1), in the absence of the maximality of $A+B$, is by using splitting methods, which have received much attention in the recent past due to their applications in image recovery, signal processing, and machine learning. One of the popular iterative methods used for solving problem (1) is the forward-backward method introduced by Passty [7] in 1979 which defines a sequence $\left(x_{n}\right)$ by

$$
x_{n+1}=\left(I+r_{n} B\right)^{-1}\left(I-r_{n} A\right) x_{n}, \quad \text { for } n=1,2, \ldots,
$$

where $\left(r_{n}\right)$ is a sequence of positive numbers, $A$ and $B$ are maximal monotone operators with $D(B) \subset D(A)$, and $A$ is single valued. Since its inception, the splitting method (2) has received much attention from several authors including Tseng [8], Mercier [9], Gabay [10], and Chen [11]. The projected gradient method is in fact a special case of scheme (2). In this case, $A$ is taken as the gradient of a function and $B$ as the subdifferential of the indicator function of a closed and convex subset of a real Hilbert space.

In the case when $T:=A+B$ is maximal monotone, the most popular iterative method for solving (1) is the proximal point algorithm (PPA) which was first introduced by Martinet [12] in 1970 and later developed by Rockafellar [13]. Due to the failure of Rockafellar's PPA to converge strongly [14] for arbitrary maximal monotone operators, several authors [1523] have presented modified versions of the PPA that always converge strongly. Recently, Yao and Noor [22] proposed the contraction proximal point algorithm

$$
x_{n+1}=\alpha_{n} u+\gamma_{n} x_{n}+\delta_{n} J_{r_{n}}^{B} x_{n}+e_{n}, \quad \text { for } n=1,2, \ldots,
$$

where $B$ is a maximal monotone operator, $\left(\alpha_{n}\right),\left(\gamma_{n}\right)$, and $\left(\delta_{n}\right)$ are sequences in $(0,1)$ with $\alpha_{n}+\gamma_{n}+\delta_{n}=1$ for all $n \geq 1, J_{r_{n}}^{B}:=\left(I+r_{n} B\right)^{-1},\left(e_{n}\right)$ is a sequence of computational errors, $u \in H$ is given, and $r_{n}>0$. Algorithm (3) was used 
to find zeros of maximal monotone operators. The viscosity approximation method which is also used for finding zeros of maximal monotone operators was introduced by Takahashi [19] as a scheme that generates sequences $\left(x_{n}\right)$ by

$$
x_{n+1}=\alpha_{n} f\left(x_{n}\right)+\left(1-\alpha_{n}\right) J_{r_{n}}^{B} x_{n}, \text { for } n=1,2, \ldots,
$$

where $f$ is a contraction from a closed and convex subset $C$ of a reflexive Banach space $X, B$ is an accretive operator (hence monotone if $X$ is a Hilbert space) with the range condition $\overline{D(B)} \subset C \subset \cap_{r>0} R(I+r B),\left(\alpha_{n}\right)$ is a sequence in $(0,1), J_{r_{n}}^{B}:=$ $\left(I+r_{n} B\right)^{-1}$, and $r_{n}>0$. Under appropriate conditions, strong convergence of scheme (4) is obtained.

Recently, the forward-backward splitting method (2), the contraction proximal point algorithm (3), and the viscosity approximation method (4) were combined to obtain the algorithm

$$
\begin{array}{r}
x_{n+1}=\alpha_{n} f\left(x_{n}\right)+\gamma_{n} x_{n}+\delta_{n} J_{r_{n}}^{B}\left(I-r_{n} A\right) x_{n}+e_{n}, \\
\text { for } n=1,2, \ldots,
\end{array}
$$

where $A: D(A)=H \rightarrow H$ is a $\beta$-cocoercive operator, $B$ : $D(B) \subset H \rightarrow H$ is a maximal monotone operator, $\left(\alpha_{n}\right),\left(\gamma_{n}\right)$, and $\left(\delta_{n}\right)$ are sequences in $(0,1)$ with $\alpha_{n}+\gamma_{n}+\delta_{n}=1$ for all $n \geq 1, J_{r_{n}}^{B}:=\left(I+r_{n} B\right)^{-1},\left(e_{n}\right)$ is a sequence of computational errors, $u \in H$ is given, $f: H \rightarrow H$ is a contraction, and $r_{n}>0$. They were able to prove that the sequence generated by (5) converges strongly to the unique fixed point of the operator $P_{S} f$, where $S:=(A+B)^{-1} 0$. Note that when $e_{n}=0$ for all $n \geq 1$, then it suffices to derive strong convergence of (5) when $f: C \rightarrow C$, where $C$ is a closed and convex subset of $H$, and $B: D(B) \subset C \rightarrow H$. Note that, in the case when $e_{n} \neq 0$, if $x_{n} \in C$ for a given $n \geq 1$, the $(n+1)$ th iterate $x_{n+1}$ may fail to be in $C$. That is, algorithm (5) is not well defined if $f: C \rightarrow C$.

It is worthy of note that Noor's algorithm (3) excludes the important case $\delta_{n} \in[1,2)$, the overrelaxed case. However, the overrelaxed factor may indeed speed up the rate of the algorithm (see [24]). That is why Wang and Cui [25] investigated the convergence of sequences generated by

$$
x_{n+1}=\alpha_{n} u+\gamma_{n} x_{n}+\delta_{n} J_{r_{n}}^{B} x_{n}+e_{n}, \text { for } n=1,2, \ldots,
$$

for $\left(\alpha_{n}\right) \subset(0,1),\left(\gamma_{n}\right) \subset(-1,1)$, and $\left(\delta_{n}\right) \subset(0,2)$ with $\alpha_{n}+\gamma_{n}+\delta_{n}=1$ for all $n \geq 1$. A natural question thus arises: is it possible to relax the conditions on $\left(\gamma_{n}\right)$ and $\left(\delta_{n}\right)$ used in algorithm (5) further? Our purpose in this paper is to affirmatively answer this question. Furthermore, we prove a strong convergence result associated with algorithm

$$
\begin{array}{r}
x_{n+1}=\alpha_{n} f\left(x_{n}\right)+\gamma_{n} x_{n}+\delta_{n} J_{r_{n}}^{B}\left(I-r_{n} A\right) x_{n}+e_{n}, \\
\text { for } n=1,2, \ldots,
\end{array}
$$

for $\left(\alpha_{n}\right) \subset(0,1),\left(\gamma_{n}\right) \subset(-3 / 2,1)$, and $\left(\delta_{n}\right) \subset(0,3 / 2)$ with $\alpha_{n}+\gamma_{n}+\delta_{n}=1$ for all $n \geq 1$, for the case when the sequence of error terms is square summable in norm. Our main result improves and refines similar results in the literature by using fewer conditions to derive strong convergence of (7). In addition, our results generalize many results in the literature such as [25, Theorem 1], [26, Theorem 1], and [27, Theorem $1]$.

\section{Preliminary Results}

Let $H$ be a real Hilbert space endowed with the inner product $\langle\cdot, \cdot\rangle$ and norm $\|\cdot\|$. A map $T: H \rightarrow H$ is called a Lipschitz mapping if there exists $L \geq 0$ such that $\|T(x)-T(y)\| \leq L \| x-$ $y \|$ for all $x, y \in H$. The number $L$ associated with $T$ is called a Lipschitz constant. If $L<1$, we say that $T$ is a contraction, and $T$ is called nonexpansive if $L=1$. The set of fixed points of $T$ is given by $F(T)=\{x \in C: T(x)=x\}$. It is well known that if $T$ is nonexpansive, then $I-T$ is demiclosed at zero and $F(T)$ is closed and convex; see [28]. Given an operator $T: H \rightarrow H$, we say that $I-T$ is demiclosed at zero if, for any sequence $\left(x_{n}\right)$ in $H$, the following implication holds:

$$
\left.\begin{array}{r}
x_{n} \rightarrow x \\
(I-T) x_{n} \longrightarrow 0
\end{array}\right\} \Longrightarrow x \in \operatorname{Fix}(T) \text {. }
$$

Here, $x_{n} \rightarrow x$ means that $\left(x_{n}\right)$ converges weakly to $x$ and $x_{n} \rightarrow x$ is used to indicate that $\left(x_{n}\right)$ converges strongly to $x$. These notations will be used in the sequel.

For a nonempty closed and convex subset $F$ of $H$, the metric projection (nearest point mapping) $P_{F}: H \rightarrow F$ is defined as follows: given $x \in H, P_{F} x$ is the unique point in $F$ having the property

$$
\left\|x-P_{F} x\right\|=\inf _{y \in F}\|x-y\| .
$$

Note that the projection operator is firmly nonexpansive and has the following characterization which will be used in this paper: for any $x \in H$,

$$
\left\langle x-P_{F} x, z-P_{F} x\right\rangle \leq 0, \quad \forall z \in F .
$$

Recall that an operator $T: H \rightarrow H$ is said to be firmly nonexpansive if for every $x, y \in H$

$$
\|T x-T y\|^{2} \leq\|x-y\|^{2}-\|(I-T) x-(I-T) y\|^{2} .
$$

It is clear that every firmly nonexpansive map is nonexpansive. A single-valued operator $A: D(A) \subset H \rightarrow H$ is called $\beta$-inverse strongly monotone $(\beta$-cocoercive) for a positive number $\beta$ if

$$
\langle A x-A y, x-y\rangle \geq \beta\|A x-A y\|^{2} \quad \forall x, y \in D(A) .
$$

It is known that, for a $\beta$-inverse strongly monotone mapping $A$, the map $T:=I-r A$ is nonexpansive for all $r \in(0,2 \beta)$. A (possibly set-valued) nonlinear operator $A: D(A) \subset H \rightarrow H$ is said to be monotone if

$$
\begin{aligned}
\left\langle x^{\prime}-y^{\prime}, x-y\right\rangle \geq & 0 \\
& \forall x, y \in D(A), x^{\prime} \in A x, y^{\prime} \in A y .
\end{aligned}
$$

In other words, $A$ is monotone if its graph, $G(A)=\{(x, y) \in$ $H \times H: x \in D(A), y \in A x\}$, is a monotone subset of $H \times H$. A monotone operator $A$ is called maximal monotone if it is monotone and its graph is not properly contained in the graph of any other monotone operator. Note that a $\beta$ cocoercive operator is monotone. We know that $A$ is maximal 
monotone if and only if the range of $I+A$ is equal to $H$ (i.e., $R(I+A)=H$ ). If $A$ is maximal monotone and $\mu$ is a positive number, then the resolvent of $A$ is a single-valued and firmly nonexpansive operator $J_{\mu}^{A}: H \rightarrow H$ defined by $J_{\mu}^{A}(x)=(I+\mu A)^{-1}(x)$. We note that $J_{\mu}^{A}$ is everywhere defined on $H$. For more information, refer to [29].

Finally, we recall some elementary inequalities in real Hilbert spaces. For every $x, y \in H$, the inequality

$$
\|x+y\|^{2} \leq\|x\|^{2}+2\langle y, x+y\rangle
$$

holds. If $\alpha, \beta$ are any real numbers in $(0,1)$ with $\alpha+\beta=1$, then, for any $x, y \in H$,

$$
\|\alpha x+\beta y\|^{2}=\alpha\|x\|^{2}+\beta\|y\|^{2}-\alpha \beta\|x-y\|^{2} .
$$

Moreover, for any $a, b \in H$, the inequality

$$
2\|a\|\|b\| \leq \frac{1}{2}\|a\|^{2}+2\|b\|^{2}
$$

can also be proved easily.

Now, we establish two lemmas that will enable us to prove our main result.

Lemma 1. Let $A$ be a $\beta$-cocoercive operator and let $r$ be a positive real number satisfying $r \leq \beta$. Then, $T:=I-r A$ is firmly nonexpansive.

Proof. We have to show that

$$
\begin{aligned}
\|T x-T y\|^{2} & \leq\|x-y\|^{2}-\|(I-T) x-(I-T) y\|^{2} \\
& =\|x-y\|^{2}-r^{2}\|A x-A y\|^{2} .
\end{aligned}
$$

Using the definition of $T$, we have

$$
\begin{aligned}
\|T x-T y\|^{2}= & \|(x-y)-r(A x-A y)\|^{2} \\
= & \|x-y\|^{2}+r^{2}\|A x-A y\|^{2} \\
& -2 r\langle A x-A y, x-y\rangle \\
\leq & \|x-y\|^{2}+r^{2}\|A x-A y\|^{2} \\
& -2 r \beta\|A x-A y\|^{2},
\end{aligned}
$$

where the inequality follows from the fact that $A$ is $\beta$ cocoercive. Since $r \leq \beta$, it follows that $-r \beta \leq-r^{2}$ and inequality (17) follows at once.

Lemma 2. Let $T$ and $S$ be firmly nonexpansive mappings. Then,

$$
\begin{aligned}
\|T S x-T S y\|^{2} \leq & \|x-y\|^{2} \\
& -\frac{1}{2}\|(I-T S) x-(I-T S) y\|^{2} .
\end{aligned}
$$

In particular, TS is nonexpansive.
Proof. From the firmly nonexpansive property of $T$, we get

$$
\begin{aligned}
& \|T S x-T S y\|^{2} \leq\|S x-S y\|^{2}-\|(I-T) S x \\
& -(I-T) S y\left\|^{2} \leq\right\| x-y\left\|^{2}-\right\|(I-S) x \\
& -(I-S) y\left\|^{2}-\right\|[(I-T S) x-(I-T S) y] \\
& -[(I-S) x-(I-S) y]\left\|^{2}=\right\| x-y \|^{2} \\
& -\|(I-T S) x-(I-T S) y\|^{2}-2 \|(I-S) x \\
& -(I-S) y \|^{2}+2\langle(I-T S) x \\
& -(I-T S) y,(I-S) x-(I-S) y\rangle,
\end{aligned}
$$

where the second inequality follows from the fact that $S$ is firmly nonexpansive. Using (16) with $a=(I-T S) x-(I-T S) y$ and $b=(I-S) x-(I-S) y$, we get

$$
\begin{aligned}
\|T S x-T S y\|^{2} \leq & \|x-y\|^{2} \\
& -\|(I-T S) x-(I-T S) y\|^{2} \\
& -2\|(I-S) x-(I-S) y\|^{2} \\
& +\frac{1}{2}\|(I-T S) x-(I-T S) y\|^{2} \\
& +2\|(I-S) x-(I-S) y\|^{2} \\
= & \|x-y\|^{2} \\
& -\frac{1}{2}\|(I-T S) x-(I-T S) y\|^{2} .
\end{aligned}
$$

The proof is complete.

Remark 3. Let $B$ be maximal monotone and let $A$ be a $\beta$ cocoercive mapping with $\beta>0$. Taking $U=J_{r}^{B}$ and $V=$ $(I-r A)$ for some $r \leq \beta$, both $U$ and $V$ are firmly nonexpansive (see Lemma 1). In addition, if $S:=\operatorname{Fix}\left(J_{r}^{B}(I-r A)\right) \neq \emptyset$, then, for any $z \in S$ and $x \in D(A)$, we obtain from Lemma 2 that

$$
\begin{aligned}
\left\|J_{r}^{B}(I-r A) x-z\right\|^{2} \leq & \|x-z\|^{2} \\
& -\frac{1}{2}\left\|x-J_{r}^{B}(I-r A) x\right\|^{2} .
\end{aligned}
$$

We next recall some lemmas that will be useful in proving our main results. In the next two lemmas, it is assumed that $A$ is a $\beta$-cocoercive mapping with $\beta>0$ and $B$ is maximal monotone.

Lemma 4. If $\lambda$ and $\mu$ are any two positive real numbers, then

$$
\begin{gathered}
\left\|J_{\lambda}^{B}(I-\lambda A) x-J_{\mu}^{B}(I-\mu A) x\right\| \\
\quad \leq\left|1-\frac{\lambda}{\mu}\right|\left\|x-J_{\mu}^{B}(I-\mu A) x\right\|
\end{gathered}
$$

holds for any $x \in H$. 
The proof of Lemma 4 can be reproduced easily.

Lemma 5 (see López et al. [3]). If $\lambda$ and $\mu$ are two positive real numbers such that $\mu \geq \lambda$, then

$$
\left\|x-J_{\lambda}^{B}(I-\lambda A) x\right\| \leq 2\left\|x-J_{\mu}^{B}(I-\mu A) x\right\|
$$

holds for any $x \in H$.

The next two lemmas are important in showing that under suitable assumptions our sequence $\left(x_{n}\right)$ generated by (7) is bounded. The proofs can be reproduced by following some ideas of [26].

Lemma 6. Let $\left(s_{n}\right)$ be a sequence of nonnegative real numbers satisfying

$$
s_{n+1} \leq\left(1-\alpha_{n}\right)\left(1+\epsilon_{n}\right) s_{n}+\alpha_{n} K+\xi_{n}
$$

for some $K>0$, where $\left(\alpha_{n}\right) \subset(0,1)$ and $\left(\epsilon_{n}\right),\left(\xi_{n}\right) \in \ell^{1}$ are sequences of positive real numbers. Then, $\left(s_{n}\right)$ is bounded.

Lemma 7. Let $\left(s_{n}\right)$ be a sequence of nonnegative real numbers satisfying

$$
s_{n+1} \leq\left(1-\alpha_{n}\right)\left(1+\epsilon_{n}\right) s_{n}+\alpha_{n} K_{1}+\epsilon_{n} K_{2},
$$

where $K_{1}, K_{2}$ are positive constants, $\left(\alpha_{n}\right)$ is a sequence in $(0,1)$, and $\epsilon_{n} \in[0, \infty)$ with $\epsilon_{n}\left(3-2 \alpha_{n}\right) \leq \alpha_{n}$ for all $n \geq 0$. Then, $\left(s_{n}\right)$ is bounded.

The last two lemmas will be vital in deducing strong convergence of the sequence generated by (7).

Lemma 8 (see $\mathrm{Xu}[20])$. Let $\left(s_{n}\right)$ be a sequence of nonnegative real numbers satisfying

$$
s_{n+1} \leq\left(1-a_{n}\right) s_{n}+a_{n} b_{n}+c_{n}, \quad n \geq 0,
$$

where $\left(a_{n}\right),\left(b_{n}\right)$, and $\left(c_{n}\right)$ satisfy the following conditions: (i) $\left(a_{n}\right) \subset[0,1]$, with $\sum_{n=0}^{\infty} a_{n}=\infty$, (ii) $c_{n} \geq 0$ for all $n \geq 0$ with $\sum_{n=0}^{\infty} c_{n}<\infty$, and (iii) $\lim \sup _{n \rightarrow \infty} b_{n} \leq 0$. Then, $\lim _{n \rightarrow \infty} s_{n}=$ 0 .

Lemma 9 (see Maingé [30]). Let $\left(s_{k}\right)$ be a sequence of real numbers that does not decrease at infinity, in the sense that there exists a subsequence $\left(s_{k_{j}}\right)$ of $\left(s_{k}\right)$ such that $s_{k_{j}}<s_{k_{j}+1}$ for all $j \geq 0$. Define an integer sequence $\left(m_{k}\right)_{k \geq k_{0}}$ as

$$
m_{k}=\max \left\{k_{0} \leq l \leq k: s_{l}<s_{l+1}\right\} .
$$

Then, $m_{k} \rightarrow \infty$ as $k \rightarrow \infty$ and for all $k \geq k_{0}$

$$
\max \left\{s_{m_{k}}, s_{k}\right\} \leq s_{m_{k}+1} .
$$

\section{Main Results}

For any $r \in(0, \beta]$, the map $J_{r}^{B}(I-r A)$, being the composition of nonexpansive maps, is nonexpansive. Since the fixed point set (if it is not empty) of a nonexpansive map is closed and convex, it follows that if $S:=(A+B)^{-1}(0) \neq \emptyset$, then $S$ is closed and convex. Therefore, the map $P_{S}$ is well defined and nonexpansive. Also, for any closed and convex set $C$, the map $P_{S} f: C \rightarrow S$ is a $\kappa$-contraction whenever $f: C \rightarrow C$ is a $\kappa$-contraction. This information will be used in this section.

Theorem 10. Let $H$ be a real Hilbert space and let $f: H \rightarrow H$ be a $\kappa$-contraction with $\kappa<1 / 2$. Assume that $A: D(A)=$ $H \rightarrow H$ is a $\beta$-cocoercive operator and $B: D(B) \subset H \rightarrow H$ is a maximal monotone operator with $S:=(A+B)^{-1}(0) \neq \emptyset$. For $x_{0} \in H$, let $\left(x_{n}\right)$ be a sequence generated by (7) with $\alpha_{n} \in(0,1)$, $\gamma_{n} \in(-3 / 2,1)$, and $\delta_{n} \in(0,3 / 2)$ satisfying $\alpha_{n}+\gamma_{n}+\delta_{n}=1$, $r_{n} \in(0, \beta]$, and $\left(e_{n}\right)$ is a sequence of errors in $H$. Then, $\left(x_{n}\right)$ converges strongly to the unique fixed point $z$ of $P_{S} f$, provided that

(i) $\lim _{n \rightarrow \infty} \alpha_{n}=0$ and $\sum_{n=0}^{\infty} \alpha_{n}=\infty$,

(ii) $\liminf _{n \rightarrow \infty} r_{n}>0$,

(iii) $0<\liminf _{n \rightarrow \infty} \delta_{n} \leq \lim \sup _{n \rightarrow \infty} \delta_{n}<3 / 2$,

(iv) $\left\|e_{n}\right\| \leq \eta_{n}\left\|x_{n}-J_{r_{n}}^{B}\left(I-r_{n} A\right) x_{n}\right\|$ with $\sum_{n=0}^{\infty} \eta_{n}^{2}<\infty$.

Proof. Let us denote $\rho_{n}:=\delta_{n}\left(1-\alpha_{n}\right)^{-1}$ and $y_{n}:=\left(1-\rho_{n}\right) x_{n}+$ $\rho_{n} J_{r_{n}}^{B}\left(I-r_{n} A\right) x_{n}$. Then, from (7), $x_{n+1}=\alpha_{n} f\left(x_{n}\right)+\left(1-\alpha_{n}\right) y_{n}+$ $e_{n}$. In order to prove that $\left(x_{n}\right)$ is bounded, we note that, from the condition $\alpha_{n} \rightarrow 0$ as $n \rightarrow \infty$, one may assume without loss of generality that $\alpha_{n}<1 / 2$ for all $n$. If $z$ is the unique fixed point of $P_{S} f$, then $z \in S$ and from (15) we have

$$
\begin{aligned}
&\left\|x_{n+1}-z\right\|^{2} \\
&=\left\|\alpha_{n}\left(f\left(x_{n}\right)-z+e_{n}\right)+\left(1-\alpha_{n}\right)\left(y_{n}-z+e_{n}\right)\right\|^{2} \\
&= \alpha_{n}\left\|f\left(x_{n}\right)-z+e_{n}\right\|^{2}+\left(1-\alpha_{n}\right)\left\|y_{n}-z+e_{n}\right\|^{2} \\
& \quad-\alpha_{n}\left(1-\alpha_{n}\right)\left\|f\left(x_{n}\right)-y_{n}\right\|^{2} \\
& \leq \alpha_{n}\left\|f\left(x_{n}\right)-z+e_{n}\right\|^{2}+\left(1-\alpha_{n}\right)\left\|y_{n}-z+e_{n}\right\|^{2} .
\end{aligned}
$$

To estimate $\left\|y_{n}-z+e_{n}\right\|^{2}$, let us first observe that

$$
\begin{aligned}
& \left\|y_{n}-z\right\|^{2}=\left\|\left(x_{n}-z\right)+\rho_{n}\left(J_{r_{n}}^{B}\left(I-r_{n} A\right) x_{n}-x_{n}\right)\right\|^{2} \\
& \quad=\left\|x_{n}-z\right\|^{2}+\rho_{n}^{2}\left\|J_{r_{n}}^{B}\left(I-r_{n} A\right) x_{n}-x_{n}\right\|^{2} \\
& \quad-\rho_{n}\left[2\left\langle x_{n}-z, x_{n}-J_{r_{n}}^{B}\left(I-r_{n} A\right) x_{n}\right\rangle\right]=\| x_{n} \\
& \quad-z\left\|^{2}+\rho_{n}^{2}\right\| J_{r_{n}}^{B}\left(I-r_{n} A\right) x_{n}-x_{n} \|^{2}-\rho_{n}\left[\left\|x_{n}-z\right\|^{2}\right. \\
& \quad+\left\|x_{n}-J_{r_{n}}^{B}\left(I-r_{n} A\right) x_{n}\right\|^{2} \\
& \left.\quad-\left\|J_{r_{n}}^{B}\left(I-r_{n} A\right) x_{n}-z\right\|^{2}\right]=\left(1-\rho_{n}\right)\left\|x_{n}-z\right\|^{2} \\
& \quad+\rho_{n}\left\|J_{r_{n}}^{B}\left(I-r_{n} A\right) x_{n}-z\right\|^{2}-\rho_{n}\left(1-\rho_{n}\right) \| x_{n} \\
& \quad-J_{r_{n}}^{B}\left(I-r_{n} A\right) x_{n} \|^{2} .
\end{aligned}
$$


Making use of inequality (22), we obtain

$$
\begin{aligned}
\left\|y_{n}-z\right\|^{2} \leq & \left\|x_{n}-z\right\|^{2} \\
& -\rho_{n}\left(\frac{3}{2}-\rho_{n}\right)\left\|x_{n}-J_{r_{n}}^{B}\left(I-r_{n} A\right) x_{n}\right\|^{2} .
\end{aligned}
$$

Therefore, it follows that

$$
\begin{aligned}
& \left\|y_{n}-z+e_{n}\right\|^{2}=\left\|y_{n}-z\right\|^{2}+\left\|e_{n}\right\|^{2}+2\left\langle y_{n}-z, e_{n}\right\rangle \\
& \leq\left\|y_{n}-z\right\|^{2}+\left\|e_{n}\right\|^{2}+\rho_{n}\left(3-2 \rho_{n}\right) \frac{\left\|e_{n}\right\|^{2}}{4 \eta_{n}^{2}} \\
& +\frac{4 \eta_{n}^{2}}{\rho_{n}\left(3-2 \rho_{n}\right)}\left\|y_{n}-z\right\|^{2}=\left(1+\frac{4 \eta_{n}^{2}}{\rho_{n}\left(3-2 \rho_{n}\right)}\right) \\
& \cdot\left(\left\|y_{n}-z\right\|^{2}+\rho_{n}\left(3-2 \rho_{n}\right) \frac{\left\|e_{n}\right\|^{2}}{4 \eta_{n}^{2}}\right) .
\end{aligned}
$$

Note that condition (iii) of the theorem is equivalent to $0<$ $\liminf _{n \rightarrow \infty} \rho_{n} \leq \limsup _{n \rightarrow \infty} \rho_{n}<3 / 2$. Then, there exist positive real numbers $\rho_{1}$ and $\rho_{2}$ such that $\rho_{1} \leq \rho_{n} \leq \rho_{2}<3 / 2$. From this condition, we derive $4 / \rho_{n}\left(3-2 \rho_{n}\right) \leq C$ for some positive constant $C$. Denote $\epsilon_{n}:=\eta_{n}^{2} C$. Since $\left(\eta_{n}^{2}\right) \in \ell^{1}$, it follows that $\left(\epsilon_{n}\right) \in \ell^{1}$ as well. Therefore, from (32) and condition (iv) of the theorem, we have

$$
\begin{aligned}
& \left\|y_{n}-z+e_{n}\right\|^{2} \leq\left(1+\epsilon_{n}\right)\left(\left\|x_{n}-z\right\|^{2}\right. \\
& \left.\quad-\frac{\rho_{n}\left(3-2 \rho_{n}\right)}{4}\left\|x_{n}-J_{r_{n}}^{B}\left(I-r_{n} A\right) x_{n}\right\|^{2}\right) .
\end{aligned}
$$

On the other hand, by similar arguments as above, we have

$$
\begin{aligned}
& \left\|f\left(x_{n}\right)-z+e_{n}\right\|^{2}=\left\|f\left(x_{n}\right)-z\right\|^{2}+\left\|e_{n}\right\|^{2} \\
& +2\left\langle f\left(x_{n}\right)-z, e_{n}\right\rangle \leq\left\|f\left(x_{n}\right)-z\right\|^{2}+\left\|e_{n}\right\|^{2} \\
& +\rho_{n}\left(3-2 \rho_{n}\right) \frac{\left\|e_{n}\right\|^{2}}{4 \eta_{n}^{2}}+\frac{4 \eta_{n}^{2}}{\rho_{n}\left(3-2 \rho_{n}\right)}\left\|f\left(x_{n}\right)-z\right\|^{2} \\
& \quad\left(1+\frac{4 \eta_{n}^{2}}{\rho_{n}\left(3-2 \rho_{n}\right)}\right) \\
& \quad\left[\left\|f\left(x_{n}\right)-z\right\|^{2}+\rho_{n}\left(3-2 \rho_{n}\right) \frac{\left\|e_{n}\right\|^{2}}{4 \eta_{n}^{2}}\right],
\end{aligned}
$$

where the inequality follows from (16). Moreover, using the property that $f$ is a $\kappa$-contraction, we get

$$
\begin{aligned}
\left\|f\left(x_{n}\right)-z\right\|^{2}= & \left\|f\left(x_{n}\right)-f(z)\right\|^{2}+\|f(z)-z\|^{2} \\
& +2\left\langle f\left(x_{n}\right)-f(z), f(z)-z\right\rangle \\
\leq & 2\left\|f\left(x_{n}\right)-f(z)\right\|^{2}+2\|f(z)-z\|^{2} \\
\leq & 2 \kappa\left\|x_{n}-z\right\|^{2}+2\|f(z)-z\|^{2} .
\end{aligned}
$$

Since $\left\|e_{n}\right\| \leq \eta_{n}\left\|x_{n}-J_{r_{n}}^{B}\left(I-r_{n} A\right) x_{n}\right\|$, it follows that

$$
\begin{aligned}
& \left\|f\left(x_{n}\right)-z+e_{n}\right\|^{2} \\
& \quad \leq\left(1+\epsilon_{n}\right)\left[2 \kappa\left\|x_{n}-z\right\|^{2}+2\|f(z)-z\|^{2}\right] \\
& \quad+\left(1+\epsilon_{n}\right) \frac{\rho_{n}\left(3-2 \rho_{n}\right)}{4}\left\|x_{n}-J_{r_{n}}^{B}\left(I-r_{n} A\right) x_{n}\right\|^{2} .
\end{aligned}
$$

Combining this last inequality with (34), it follows from (30) that

$$
\begin{aligned}
& \left\|x_{n+1}-z\right\|^{2} \leq\left(1-\alpha_{n}(1-2 \kappa)\right)\left(1+\epsilon_{n}\right)\left\|x_{n}-z\right\|^{2} \\
& +\alpha_{n} K+\epsilon_{n} K-\left(1-2 \alpha_{n}\right)\left(1+\epsilon_{n}\right) \\
& \quad \frac{\rho_{n}\left(3-2 \rho_{n}\right)}{4}\left\|x_{n}-J_{r_{n}}^{B}\left(I-r_{n} A\right) x_{n}\right\|^{2},
\end{aligned}
$$

where $K=2\|f(z)-z\|^{2}$. Since $\alpha_{n}<1 / 2$ for very large $n$, by our assumption, the inequality above reduces to

$$
\begin{aligned}
\left\|x_{n+1}-z\right\|^{2} \leq & \left(1-\alpha_{n}(1-2 \kappa)\right)\left(1+\epsilon_{n}\right)\left\|x_{n}-z\right\|^{2} \\
& +\alpha_{n} K+\epsilon_{n} K .
\end{aligned}
$$

Applying Lemma 6 with $s_{n}:=\left\|x_{n}-z\right\|^{2}$ and $\xi_{n}:=\epsilon_{n} K$, we derive that the sequence $\left(x_{n}-z\right)$ is bounded. Therefore, $\left(x_{n}\right)$ is bounded.

We next show that

$$
\begin{aligned}
\left\|x_{n+1}-z\right\|^{2} \leq & \left(1-\alpha_{n}(1-2 \kappa)\right)\left\|x_{n}-z\right\|^{2}+\alpha_{n} d_{n} \\
& +\epsilon_{n} M \\
& -L\left(1-\alpha_{n}\right)\left\|x_{n}-J_{r_{n}}^{B}\left(I-r_{n} A\right) x_{n}\right\|^{2}
\end{aligned}
$$

holds for some positive constants $L$ and $M$, where $d_{n}$ denotes

$$
d_{n}:=2\left\langle f(z)-z+e_{n}, x_{n+1}-z\right\rangle+\left(\alpha_{n}+\left\|e_{n}\right\|\right) M .
$$

To this end, we first note that the condition $\rho_{n}<3 / 2$ and (32) imply that

$$
\left\|y_{n}-z\right\| \leq\left\|x_{n}-z\right\| \text {. }
$$

Therefore, from the equality $x_{n+1}=\alpha_{n} f\left(x_{n}\right)+\left(1-\alpha_{n}\right) y_{n}+e_{n}$ and using the fact that $f$ is a $\kappa$-contraction, we obtain

$$
\begin{aligned}
\left\|x_{n+1}-z\right\| \leq & \alpha_{n}\left\|f\left(x_{n}\right)-z\right\|+\left(1-\alpha_{n}\right)\left\|y_{n}-z\right\| \\
& +\left\|e_{n}\right\| \\
\leq & \alpha_{n}\left[\left\|f\left(x_{n}\right)-f(z)\right\|+\|f(z)-z\|\right] \\
& +\left(1-\alpha_{n}\right)\left\|x_{n}-z\right\|+\left\|e_{n}\right\| \\
\leq & \alpha_{n}\left[\kappa\left\|x_{n}-z\right\|+\|f(z)-z\|\right] \\
& +\left(1-\alpha_{n}\right)\left\|x_{n}-z\right\|+\left\|e_{n}\right\| \\
= & \left(1-\alpha_{n}(1-\kappa)\right)\left\|x_{n}-z\right\| \\
& +\alpha_{n}\|f(z)-z\|+\left\|e_{n}\right\| \\
\leq & \left\|x_{n}-z\right\|+\alpha_{n}\|f(z)-z\|+\left\|e_{n}\right\| .
\end{aligned}
$$


On the other hand, it follows from (14) that

$$
\begin{aligned}
\| x_{n+1} & -z \|^{2} \\
= & \left\|\left(1-\alpha_{n}\right)\left(y_{n}-z+e_{n}\right)+\alpha_{n}\left(f\left(x_{n}\right)-z+e_{n}\right)\right\|^{2} \\
\leq & \left(1-\alpha_{n}\right)\left\|y_{n}-z+e_{n}\right\|^{2} \\
& +2 \alpha_{n}\left\langle f\left(x_{n}\right)-z+e_{n}, x_{n+1}-z\right\rangle \\
= & \left(1-\alpha_{n}\right)\left\|y_{n}-z+e_{n}\right\|^{2} \\
& +2 \alpha_{n}\left\langle f(z)-z+e_{n}, x_{n+1}-z\right\rangle \\
& +2 \alpha_{n}\left\langle f\left(x_{n}\right)-f(z), x_{n+1}-z\right\rangle \\
\leq & \left(1-\alpha_{n}\right)\left\|y_{n}-z+e_{n}\right\|^{2} \\
& +2 \alpha_{n}\left\langle f(z)-z+e_{n}, x_{n+1}-z\right\rangle \\
& +2 \alpha_{n}\left\|f\left(x_{n}\right)-f(z)\right\|\left\|x_{n+1}-z\right\| .
\end{aligned}
$$

Again, using the fact that $f$ is a $\kappa$-contraction with $\kappa<1 / 2$, we have

$$
\begin{aligned}
& \left\|x_{n+1}-z\right\|^{2} \leq\left(1-\alpha_{n}\right)\left\|y_{n}-z+e_{n}\right\|^{2} \\
& \quad+2 \alpha_{n}\left\langle f(z)-z+e_{n}, x_{n+1}-z\right\rangle \\
& \quad+2 \alpha_{n} \kappa\left\|x_{n}-z\right\|\left\|x_{n+1}-z\right\| \\
& \quad \leq\left(1-\alpha_{n}\right)\left\|y_{n}-z+e_{n}\right\|^{2} \\
& \quad+2 \alpha_{n}\left\langle f(z)-z+e_{n}, x_{n+1}-z\right\rangle \\
& \quad+2 \alpha_{n} \kappa\left\|x_{n}-z\right\|\left[\left\|x_{n}-z\right\|+\alpha_{n}\|f(z)-z\|+\left\|e_{n}\right\|\right] \\
& \quad \leq\left(1-\alpha_{n}\right)\left\|y_{n}-z+e_{n}\right\|^{2} \\
& +2 \alpha_{n}\left\langle f(z)-z+e_{n}, x_{n+1}-z\right\rangle+2 \alpha_{n} \kappa\left\|x_{n}-z\right\|^{2} \\
& +\alpha_{n}\left(\alpha_{n}+\left\|e_{n}\right\|\right) M,
\end{aligned}
$$

where $M=(1+\|f(z)-z\|) \sup _{n}\left\|x_{n}-z\right\|$. Combining this inequality with (34), we obtain

$$
\begin{aligned}
& \left\|x_{n+1}-z\right\|^{2} \leq\left(1-\alpha_{n}(1-2 \kappa)\right)\left(1+\epsilon_{n}\right)\left\|x_{n}-z\right\|^{2} \\
& +\alpha_{n}\left(\alpha_{n}+\left\|e_{n}\right\|\right) M-\left(1-\alpha_{n}\right)\left(1+\epsilon_{n}\right) \\
& \quad \cdot \frac{\rho_{n}\left(3-2 \rho_{n}\right)}{4}\left\|x_{n}-J_{r_{n}}^{B}\left(I-r_{n} A\right) x_{n}\right\|^{2} \\
& +2 \alpha_{n}\left\langle f(z)-z+e_{n}, x_{n+1}-z\right\rangle .
\end{aligned}
$$

Using condition (iii) of the theorem and the boundedness of $\left(x_{n}\right)$, we readily get $(40)$.

Now, from $\left(\epsilon_{n}\right) \in \ell^{1}$, we can find $v>0$ such that

$$
v:=\lim _{n \rightarrow \infty} \sum_{k=1}^{n} \epsilon_{k} .
$$

Therefore, if we denote $s_{n}:=\left\|x_{n}-z\right\|^{2}+t_{n} M$, where $\left(t_{n}\right)$ is a nonnegative sequence that converges to zero and is defined by

$$
t_{n}=v-\sum_{k=1}^{n-1} \epsilon_{k},
$$

then $\left(s_{n}\right)$ converges to zero strongly if and only if $\left(\left\|x_{n}-z\right\|\right)$ does. In addition, we rewrite inequality (40) in the form

$$
\begin{aligned}
s_{n+1} \leq & \left(1-\alpha_{n}(1-2 \kappa)\right) s_{n}+\alpha_{n} b_{n} \\
& -L\left(1-\alpha_{n}\right)\left\|x_{n}-J_{r_{n}}^{B}\left(I-r_{n} A\right) x_{n}\right\|^{2},
\end{aligned}
$$

where $b_{n}$ denotes

$$
\begin{aligned}
b_{n}:= & 2\left\langle f(z)-z+e_{n}, x_{n+1}-z\right\rangle \\
& +\left(\alpha_{n}+t_{n}+\left\|e_{n}\right\|\right) M .
\end{aligned}
$$

The next step is to show that $\left(s_{n}\right)$ converges strongly to zero. We achieve this by considering two possible cases on the sequence $\left(s_{n}\right)$.

Case 1 . We assume that $\left(s_{n}\right)$ is eventually decreasing (i.e., there exists $n_{0} \geq 1$ such that $\left(s_{n}\right)$ is decreasing for all $\left.n \geq n_{0}\right)$. Then, $\left(s_{n}\right)$ converges and rearranging terms in (49) we obtain

$$
\begin{aligned}
& L\left(1-\alpha_{n}\right)\left\|x_{n}-J_{r_{n}}^{B}\left(I-r_{n} A\right) x_{n}\right\|^{2} \\
& \quad \leq\left(1-\alpha_{n}(1-2 \kappa)\right) s_{n}-s_{n+1}+\alpha_{n} b_{n} .
\end{aligned}
$$

Since $\left(x_{n}\right)$ is bounded, we pass to the limit in the above inequality to get

$$
\lim _{n \rightarrow \infty}\left\|x_{n}-J_{r_{n}}^{B}\left(I-r_{n} A\right) x_{n}\right\|=0 .
$$

Take a subsequence $\left(x_{n_{k}}\right)$ of $\left(x_{n}\right)$ converging weakly to $\widehat{x}$ such that

$$
\begin{aligned}
& \limsup _{n \rightarrow \infty}\left\langle f(z)-z, x_{n}-z\right\rangle \\
& \quad=\lim _{k \rightarrow \infty}\left\langle f(z)-z, x_{n_{k}}-z\right\rangle=\langle f(z)-z, \widehat{x}-z\rangle .
\end{aligned}
$$

Note that, from (7), we derive the inequality

$$
\begin{aligned}
\left\|x_{n+1}-x_{n}\right\| \leq & \alpha_{n}\left\|f\left(x_{n}\right)-x_{n}\right\| \\
& +\delta_{n}\left\|x_{n}-J_{r_{n}}^{B}\left(I-r_{n} A\right) x_{n}\right\|+\left\|e_{n}\right\|,
\end{aligned}
$$

which together with (52), the boundedness of $\left(x_{n}\right)$, and $\left\|e_{n}\right\| \rightarrow 0$ as $n \rightarrow \infty$ implies that

$$
\lim _{n \rightarrow \infty}\left\|x_{n+1}-x_{n}\right\|=0 .
$$

The above limit implies that

$$
\begin{gathered}
\limsup _{n \rightarrow \infty}\left\langle f(z)-z+e_{n}, x_{n+1}-z\right\rangle \\
=\langle f(z)-z, \widehat{x}-z\rangle .
\end{gathered}
$$


If $r>0$ is the lower bound of $\left(r_{n}\right)$, then we have from Lemma 5

$$
\left\|x_{n}-J_{r}^{B}(I-r A) x_{n}\right\| \leq 2\left\|x_{n}-J_{r_{n}}^{B}\left(I-r_{n} A\right) x_{n}\right\|,
$$

which together with (52) implies that

$$
\lim _{n \rightarrow \infty}\left\|x_{n}-J_{r}^{B}(I-r A) x_{n}\right\|=0 .
$$

Since $S=\operatorname{Fix}\left(J_{r_{n}}^{B}\left(I-r_{n} A\right)\right)=\operatorname{Fix}\left(J_{r}^{B}(I-r A)\right)$ for all $n \geq 1$ and $J_{r}^{B}(I-r A)$ is nonexpansive, it follows that $I-J_{r}^{B}(I-r A)$ is demiclosed at zero; see [29, page 20]. Therefore, from (58) and the property that $I-J_{r}^{B}(I-r A)$ is demiclosed at zero, we conclude that $\hat{x} \in S$. Hence, from the characterization of projections, we conclude that

$$
\limsup _{n \rightarrow \infty}\left\langle f(z)-z+e_{n}, x_{n+1}-z\right\rangle \leq 0 .
$$

By conditions (i) and (iv) of the theorem and the fact that $t_{n} \rightarrow 0$ as $n \rightarrow \infty$, it follows that

$$
\limsup _{n \rightarrow \infty} b_{n} \leq 0 \text {. }
$$

Finally, we derive from inequality (49) that

$$
s_{n+1} \leq\left(1-\alpha_{n}(1-2 \kappa)\right) s_{n}+\alpha_{n} b_{n} .
$$

The conclusion that $s_{n} \rightarrow 0$ follows from Lemma 8 .

Case 2. The sequence $\left(s_{k}\right)$ is not eventually decreasing; that is, there is a subsequence $\left(s_{k_{j}}\right)$ of $\left(s_{k}\right)$ such that $s_{k_{j}}<s_{k_{j}+1}$ for all $j \geq 0$. In this case, we define an integer sequence $\left(m_{k}\right)_{k \geq k_{0}}$ as in Lemma 9. Note that the subsequence $\left(s_{m_{k}}\right)$ satisfies the condition $s_{m_{k}} \leq s_{m_{k}+1}$ for all $k \geq n_{0}$. It then follows from (49) that

$$
\begin{aligned}
& \alpha_{m_{k}}(1-2 \kappa) s_{m_{k}+1} \\
& \quad+L\left(1-\alpha_{m_{k}}\right)\left\|x_{m_{k}}-J_{r_{m_{k}}}^{B}\left(I-r_{m_{k}} A\right) x_{m_{k}}\right\|^{2} \\
& \quad \leq \alpha_{m_{k}} b_{m_{k}} .
\end{aligned}
$$

Since $2 \kappa<1, \alpha_{m_{k}} \rightarrow 0$ as $k \rightarrow \infty$, and $\left(b_{m_{k}}\right)$ is bounded, we conclude that

$$
\lim _{k \rightarrow \infty}\left\|x_{m_{k}}-J_{r_{m_{k}}}^{B}\left(I-r_{m_{k}} A\right) x_{m_{k}}\right\|=0 .
$$

Using similar arguments as in Case 1, we conclude that

$$
\limsup _{k \rightarrow \infty} b_{m_{k}} \leq 0
$$

In view of (62), we have

$$
(1-2 \kappa) s_{m_{k}+1} \leq b_{m_{k}}
$$

which implies that $s_{m_{k}+1} \rightarrow 0$ as $k \rightarrow \infty$. Since $s_{k} \leq s_{m_{k}+1}$ for all $k \geq k_{0}$ (see (29)), we also have $s_{k} \rightarrow 0$ as $k \rightarrow \infty$. Hence, $x_{k} \rightarrow z$ as $k \rightarrow \infty$, and the proof is complete.
Remark 11. Observe that if $\left(x_{n}\right)$ is bounded, then condition (iv) of Theorem 10 implies that $\sum_{n=0}^{\infty}\left\|e_{n}\right\|^{2}<\infty$. The latter condition is weaker than the condition $\sum_{n=0}^{\infty}\left\|e_{n}\right\|<$ $\infty$ used in the existing literature. In addition, we did not require the conditions $\beta_{n} \geq 1 / 2,\left(r_{n}\right) \subset(0,1)$, and $\lim \sup _{n \rightarrow \infty} r_{n}<1$. Therefore, Theorem 10 is an improvement of the aforementioned theorem.

Remark 12. Theorem 10 also improves and extends many results that exist in the literature, such as [22, Theorem 2.2], [27, Theorem 1], and [26, Theorem 1]. To see this, it is enough to take $A=0$ and $f(x)=u$ for all $x \in H$, where $u \in H$ is a given fixed vector.

Theorem 13. Let $C$ be a nonempty, closed, and convex subset of $H$ and let $f: C \rightarrow C$ be a $\kappa$-contraction with $\kappa<1 / 2$. Assume that $A: D(A)=C \rightarrow H$ is a $\beta$-cocoercive operator and $B: D(B) \subset C \rightarrow H$ is a maximal monotone operator with $S:=(A+B)^{-1}(0) \neq \emptyset$. For $x_{0} \in C$ and $e_{n}=0$ for all $n \geq 1$, let $\left(x_{n}\right)$ be a sequence generated by (7) with $\alpha_{n}, \gamma_{n}, \delta_{n} \in(0,1)$ satisfying $\alpha_{n}+\gamma_{n}+\delta_{n}=1$, and $r_{n} \in(0, \beta]$. Then, $\left(x_{n}\right)$ converges strongly to the unique fixed point $z$ of $P_{S} f$, provided that

(i) $\lim _{n \rightarrow \infty} \alpha_{n}=0$ and $\sum_{n=0}^{\infty} \alpha_{n}=\infty$,

(ii) $\liminf \operatorname{in}_{n \rightarrow \infty} r_{n}>0$,

(iii) $0<\liminf _{n \rightarrow \infty} \delta_{n}$.

Proof. In the proof of Theorem 10, we used the condition $\limsup _{n \rightarrow \infty} \delta_{n}<3 / 2$ repeatedly to conclude that $\left(3-2 \rho_{n}\right)>0$ for all $n \geq 0$, where $\rho_{n}=\delta_{n}\left(1-\alpha_{n}\right)^{-1}$. So if $\delta_{n} \in(0,1)$, then automatically $\left(3-2 \rho_{n}\right)>0$ for all $n \geq 0$.

Remark 14. We have dropped the conditions $\beta_{n} \geq 1 / 2, r_{n} \in$ $(0,1)$, and $\limsup _{n \rightarrow \infty} r_{n}<1$ used in the literature to derive strong convergence of the sequence generated by (7) under the conditions of Theorem 13. Therefore, Theorem 13 is an improvement of the aforementioned results.

Theorem 15. Let $H$ be a real Hilbert space and let $f: H \rightarrow H$ be a $\kappa$-contraction with $\kappa<1 / 2$. Assume that $A: D(A)=$ $H \rightarrow H$ is a $\beta$-cocoercive operator and $B: D(B) \subset H \rightarrow H$ is a maximal monotone operator with $S:=(A+B)^{-1}(0) \neq \emptyset$. For $x_{0} \in H$, let $\left(x_{n}\right)$ be a sequence generated by (7) with $\alpha_{n} \in(0,1)$, $\gamma_{n} \in(-3 / 2,1)$, and $\delta_{n} \in(0,3 / 2)$ satisfying $\alpha_{n}+\gamma_{n}+\delta_{n}=1$, $r_{n} \in(0, \beta]$, and $\left(e_{n}\right)$ is a sequence of errors in $H$. Then, $\left(x_{n}\right)$ converges strongly to the unique fixed point $z$ of $P_{S} f$, provided that

(i) $\lim _{n \rightarrow \infty} \alpha_{n}=0$ and $\sum_{n=0}^{\infty} \alpha_{n}=\infty$,

(ii) $\liminf _{n \rightarrow \infty} r_{n}>0$,

(iii) $0<\liminf _{n \rightarrow \infty} \delta_{n} \leq \lim \sup _{n \rightarrow \infty} \delta_{n}<3 / 2$,

(iv) $\left\|e_{n}\right\| \leq \eta_{n}\left\|x_{n}-J_{r_{n}}^{B}\left(I-r_{n} A\right) x_{n}\right\|$ with $\lim _{n \rightarrow \infty} \eta_{n}^{2} / \alpha_{n}=0$. 
Proof. Similar to the proof of Theorem 10, we assume without loss of generality that $\alpha_{n}<1 / 2$ for all $n$. Following similar steps as in the proof of Theorem 10, we derive

$$
\begin{aligned}
& \left\|x_{n+1}-z\right\|^{2} \leq\left(1-\alpha_{n}(1-2 \kappa)\right)\left(1+\epsilon_{n}\right)\left\|x_{n}-z\right\|^{2} \\
& +\alpha_{n} K+\epsilon_{n} K-\left(1-2 \alpha_{n}\right)\left(1+\epsilon_{n}\right) \\
& \quad \cdot \frac{\rho_{n}\left(3-2 \rho_{n}\right)}{4}\left\|x_{n}-J_{r_{n}}^{B}\left(I-r_{n} A\right) x_{n}\right\|^{2}
\end{aligned}
$$

where $z$ is the unique fixed point of $P_{S} f, K:=2\|f(z)-z\|^{2}$, and $\epsilon_{n}:=C \eta_{n}^{2}$ for some positive constant $C$. Since $\alpha_{n} \leq 1 / 2$ for all $n$, by our assumption, the inequality above reduces to

$$
\begin{aligned}
\left\|x_{n+1}-z\right\|^{2} \leq & \left(1-\alpha_{n}(1-2 \kappa)\right)\left(1+\epsilon_{n}\right)\left\|x_{n}-z\right\|^{2} \\
& +\alpha_{n} K+\epsilon_{n} K .
\end{aligned}
$$

It is clear from the condition $\eta_{n}^{2} / \alpha_{n} \rightarrow 0$ as $n \rightarrow 0$ that $\epsilon_{n} / \alpha_{n} \rightarrow 0$ as $n \rightarrow 0$, and we may therefore assume without loss of generality that $\epsilon_{n}\left(3-2 \alpha_{n}\right) \leq \alpha_{n}$ for all $n \geq 0$. The conclusion that $\left(x_{n}\right)$ is bounded follows on applying Lemma 7 with $s_{n}:=\left\|x_{n}-z\right\|^{2}$.

Again, similar to the proof of Theorem 10, we obtain

$$
\begin{aligned}
\left\|x_{n+1}-z\right\|^{2} \leq & \left(1-\alpha_{n}(1-2 \kappa)\right)\left\|x_{n}-z\right\|^{2}+\alpha_{n} \widehat{b}_{n} \\
& -L\left(1-\alpha_{n}\right)\left\|x_{n}-J_{r_{n}}^{B}\left(I-r_{n} A\right) x_{n}\right\|^{2},
\end{aligned}
$$

for some positive constant $L$, where $\left(\widehat{b}_{n}\right)$ is the sequence denoted by

$$
\begin{aligned}
\widehat{b}_{n}:= & 2\left\langle f(z)-z+e_{n}, x_{n+1}-z\right\rangle \\
& +\left(\alpha_{n}+\left\|e_{n}\right\|+\frac{\epsilon_{n}}{\alpha_{n}}\right) M
\end{aligned}
$$

with an appropriate constant $M>0$. We will show that $s_{n}:=$ $\left\|x_{n}-z\right\|^{2} \rightarrow 0$ as $n \rightarrow \infty$ by considering the following two cases on the sequence $\left(s_{n}\right)$.

Case 1. Assume that $\left(s_{n}\right)$ is eventually decreasing (i.e., there exists $n_{0} \geq 1$ such that $\left(s_{n}\right)$ is decreasing for all $\left.n \geq n_{0}\right)$. Then, $\left(s_{n}\right)$ converges and rearranging terms in (68) yields

$$
\begin{aligned}
& L\left(1-\alpha_{n}\right)\left\|x_{n}-J_{r_{n}}^{B}\left(I-r_{n} A\right) x_{n}\right\|^{2}+\alpha_{n}(1-2 \kappa) s_{n} \\
& \quad \leq\left(s_{n}-s_{n+1}\right)+\alpha_{n} \widehat{b}_{n},
\end{aligned}
$$

which implies that

$$
\lim _{n \rightarrow \infty}\left\|x_{n}-J_{r_{n}}^{B}\left(I-r_{n} A\right) x_{n}\right\|=0 .
$$

From this limit and Lemma 5, we obtain

$$
\lim _{n \rightarrow \infty}\left\|x_{n}-J_{r}^{B}(I-r A) x_{n}\right\|=0,
$$

where $r>0$ is the lower bound of $\left(r_{n}\right)$. Then, following similar steps as in the proof of Theorem 10, we obtain

$$
\limsup _{n \rightarrow \infty}\left\langle f(z)-z, x_{n+1}-z\right\rangle \leq 0 .
$$

As a consequence of $\eta_{n}^{2} / \alpha_{n} \rightarrow 0$ and $\alpha_{n} \rightarrow 0$ as $n \rightarrow \infty$, we derive $\left\|e_{n}\right\| \rightarrow 0$ as $n \rightarrow \infty$ and also

$$
\limsup _{n \rightarrow \infty} \widehat{b}_{n} \leq 0 \text {. }
$$

Therefore, from (68), we obtain

$$
\left\|x_{n+1}-z\right\|^{2} \leq\left(1-\alpha_{n}(1-2 \kappa)\right)\left\|x_{n}-z\right\|^{2}+\alpha_{n} \widehat{b}_{n},
$$

which together with the last limit and Lemma 8 implies that $\left\|x_{n}-z\right\| \rightarrow 0$ as $n \rightarrow \infty$.

Case 2. The sequence $\left(s_{k}\right)$ is not eventually decreasing; that is, there is a subsequence $\left(s_{k_{j}}\right)$ of $\left(s_{k}\right)$ such that $s_{k_{j}}<s_{k_{j}+1}$ for all $j \geq 0$. Again in this case we define an integer sequence $\left(m_{k}\right)_{k \geq k_{0}}$ as in Lemma 9. Note that the subsequence $\left(s_{m_{k}}\right)$ satisfies the condition $s_{m_{k}} \leq s_{m_{k}+1}$ for all $k \geq k_{0}$. It then follows from (68) that

$$
\begin{aligned}
& \left\|x_{m_{k}+1}-z\right\|^{2} \\
& \leq\left(1-\alpha_{m_{k}}(1-2 \kappa)\right)\left\|x_{m_{k}+1}-z\right\|^{2}+\alpha_{m_{k}} \widehat{b}_{m_{k}} \\
& \quad-L\left(1-\alpha_{m_{k}}\right)\left\|x_{m_{k}}-J_{r_{m_{k}}}^{B}\left(I-r_{m_{k}} A\right) x_{m_{k}}\right\|^{2},
\end{aligned}
$$

for some positive constant $L$, where $\left(\widehat{b}_{m_{k}}\right)$ is the sequence denoted by

$$
\begin{aligned}
\widehat{b}_{m_{k}}:= & 2\left\langle f(z)-z+e_{m_{k}}, x_{m_{k}+1}-z\right\rangle \\
& +\left(\alpha_{m_{k}}+\left\|e_{m_{k}}\right\|+\frac{\epsilon_{m_{k}}}{\alpha_{m_{k}}}\right) M
\end{aligned}
$$

with an appropriate constant $M>0$. Note that, from $\eta_{m_{k}}^{2} / \alpha_{m_{k}} \rightarrow 0$ and $\alpha_{m_{k}} \rightarrow 0$ as $k \rightarrow \infty$, we derive $\left\|e_{m_{k}}\right\| \rightarrow 0$ as $k \rightarrow \infty$ and

$$
\limsup _{k \rightarrow \infty} \widehat{b}_{m_{k}}=\limsup _{k \rightarrow \infty}\left\langle f(z)-z+e_{m_{k}}, x_{m_{k}+1}-z\right\rangle .
$$

Rearranging terms in (76), we obtain

$$
\begin{gathered}
L\left(1-\alpha_{m_{k}}\right)\left\|x_{m_{k}}-J_{r_{m_{k}}}^{B}\left(I-r_{m_{k}} A\right) x_{m_{k}}\right\|^{2} \\
+\alpha_{m_{k}}(1-2 \kappa) s_{m_{k}+1} \leq \alpha_{m_{k}} \widehat{b}_{m_{k}},
\end{gathered}
$$

for $s_{m_{k}+1}=\left\|x_{m_{k}+1}-z\right\|^{2}$. Taking the limit as $k \rightarrow \infty$ and noting that $\left(\widehat{b}_{m_{k}}\right)$ is bounded, we arrive at

$$
\lim _{k \rightarrow \infty}\left\|x_{m_{k}}-J_{r_{m_{k}}}^{B}\left(I-r_{m_{k}} A\right) x_{m_{k}}\right\|=0
$$

Similar to the proof of Theorem 10, we deduce that

$$
\begin{aligned}
\limsup _{k \rightarrow \infty} \widehat{b}_{m_{k}} & =\limsup _{k \rightarrow \infty}\left\langle f(z)-z+e_{m_{k}}, x_{m_{k}+1}-z\right\rangle \\
& \leq 0 .
\end{aligned}
$$


From (79), we derive

$$
(1-2 \kappa) s_{m_{k}+1} \leq \widehat{b}_{m_{k}},
$$

which together with the fact that $2 \kappa<1$ implies that $s_{m_{k}+1} \rightarrow$ 0 as $k \rightarrow \infty$. Since $s_{k} \leq s_{m_{k}+1}$ for all $k \geq n_{0}$, it follows that $s_{k} \rightarrow 0$ as $k \rightarrow \infty$. This completes the proof of the theorem.

Remark 16. If we take $A=0$ and $f(x)=u$ for all $x \in H$ in Theorem 15, where $u \in H$ is a given fixed vector, then we get an improvement and extension of [26, Theorem 2]. Strong convergence results that extend [22, Theorem 2.2] and [27, Theorem 1] and many other results associated with the contraction proximal point algorithm under the accuracy criterion given in condition (iv) of Theorem 15 can also be obtained.

\section{Competing Interests}

The author declares that there are no competing interests.

\section{Acknowledgments}

The author thanks Professor Habtu Zegeye for his valuable comments which helped to improve the presentation of this paper.

\section{References}

[1] J. Eckstein and B. F. Svaiter, "A family of projective splitting methods for the sum of two maximal monotone operators," Mathematical Programming B, vol. 111, no. 1-2, pp. 173-199, 2008.

[2] J. Eckstein and B. F. Svaiter, "General projective splitting methods for sums of maximal monotone operators," SIAM Journal on Control and Optimization, vol. 48, no. 2, pp. 787-811, 2009.

[3] G. López, V. Martín-Márquez, F. Wang, and H.-K. Xu, "Forward-backward splitting methods for accretive operators in Banach spaces," Abstract and Applied Analysis, vol. 2012, Article ID 109236, 25 pages, 2012.

[4] S. Takahashi, W. Takahashi, and M. Toyoda, "Strong convergence theorems for maximal monotone operators with nonlinear mappings in Hilbert spaces," Journal of Optimization Theory and Applications, vol. 147, no. 1, pp. 27-41, 2010.

[5] G.-J. Tang and N.-J. Huang, "Strong convergence of a splitting proximal projection method for the sum of two maximal monotone operators," Operations Research Letters, vol. 40, no. 5, pp. 332-336, 2012.

[6] R. T. Rockafellar, "On the maximality of sums of nonlinear monotone operators," Transactions of the American Mathematical Society, vol. 149, pp. 75-88, 1970.

[7] G. B. Passty, "Ergodic convergence to a zero of the sum of monotone operators in Hilbert space," Journal of Mathematical Analysis and Applications, vol. 72, no. 2, pp. 383-390, 1979.

[8] P. Tseng, "A modified forward-backward splitting method for maximal monotone mappings," SIAM Journal on Control and Optimization, vol. 38, no. 2, pp. 431-446, 2000.

[9] B. Mercier, Inéquations Variationnnelles de la Mécanique, Publications Mathématiques d'Orsay 80.01, Université Paris-Sud, Orsay, France, 1980.
[10] D. Gabay, "Applications of the method of multipliers to variational inequalities," in Augmented Lagrangian Methods: Applications to the Numerical Solution of Boundary Value Problems, M. Fortin and R. Glowinski, Eds., pp. 229-331, North-Holland Publishing, Amsterdam, The Netherlands, 1983.

[11] G. H.-G. Chen, Forward-backward splitting techniques: theory and applications [Ph.D. thesis], Department of Applied Mathematics, University of Washington, Seattle, Wash, USA, 1994.

[12] B. Martinet, "Régularisation d'inéquations variationnelles par approximations successives," Revue Francaise d'Informatique et de Recherche Opérationnelle, vol. 3, pp. 154-158, 1970.

[13] R. T. Rockafellar, "Monotone operators and the proximal point algorithm," SIAM Journal on Control and Optimization, vol. 14, no. 5, pp. 877-898, 1976.

[14] O. Güler, "On the convergence of the proximal point algorithm for convex minimization," SIAM Journal on Control and Optimization, vol. 29, no. 2, pp. 403-419, 1991.

[15] O. A. Boikanyo and G. Moroşanu, "Four parameter proximal point algorithms," Nonlinear Analysis: Theory, Methods \& Applications, vol. 74, no. 2, pp. 544-555, 2011.

[16] S. Kamimura and W. Takahashi, "Approximating solutions of maximal monotone operators in Hilbert spaces," Journal of Approximation Theory, vol. 106, no. 2, pp. 226-240, 2000.

[17] N. Lehdili and A. Moudafi, "Combining the proximal algorithm and Tikhonov regularization," Optimization, vol. 37, no. 3, pp. 239-252, 1996.

[18] M. V. Solodov and B. F. Svaiter, "Forcing strong convergence of proximal point iterations in a Hilbert space," Mathematical Programming, vol. 87, no. 1, pp. 189-202, 2000.

[19] W. Takahashi, "Approximating solutions of accretive operators by viscosity approximation methods in Banach spaces," in Applied Functional Analysis, pp. 225-243, Yokohama Publishers, Yokohama, Japan, 2007.

[20] H.-K. Xu, "Iterative algorithms for nonlinear operators," Journal of the London Mathematical Society. Second Series, vol. 66, no. 1, pp. 240-256, 2002.

[21] H.-K. Xu, "A regularization method for the proximal point algorithm," Journal of Global Optimization, vol. 36, no. 1, pp. 115$125,2006$.

[22] Y. Yao and M. A. Noor, "On convergence criteria of generalized proximal point algorithms," Journal of Computational and Applied Mathematics, vol. 217, no. 1, pp. 46-55, 2008.

[23] Y. Yao and N. Shahzad, "Strong convergence of a proximal point algorithm with general errors," Optimization Letters, vol. 6, no. 4, pp. 621-628, 2012.

[24] J. Eckstein and M. C. Ferris, "Operator-splitting methods for monotone affine variational inequalities, with a parallel application to optimal control," INFORMS Journal on Computing, vol. 10, no. 2, pp. 218-235, 1998.

[25] F. Wang and H. Cui, "Convergence of the generalized contraction-proximal point algorithm in a Hilbert space," Optimization, vol. 64, no. 4, pp. 709-715, 2015.

[26] C. Tian and F. Wang, "The contraction-proximal point algorithm with square-summable errors," Fixed Point Theory and Applications, vol. 2013, article 93, 2013.

[27] F. Wang and H. Cui, "On the contraction-proximal point algorithms with multi-parameters," Journal of Global Optimization, vol. 54, no. 3, pp. 485-491, 2012.

[28] K. Goebel and W. A. Kirk, Topics in Metric Fixed Point Theory, vol. 28, Cambridge University Press, Cambridge, UK, 1990. 
[29] G. Moroşanu, Nonlinear Evolution Equations and Applications, vol. 26 of Mathematics and Its Applications, Reidel, Dordrecht, The Netherlands, 1988.

[30] P.-E. Maingé, "Strong convergence of projected subgradient methods for nonsmooth and nonstrictly convex minimization," Set-Valued Analysis, vol. 16, no. 7-8, pp. 899-912, 2008. 


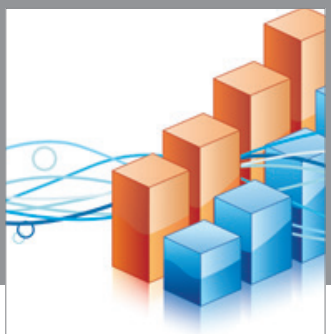

Advances in

Operations Research

vatem alat4

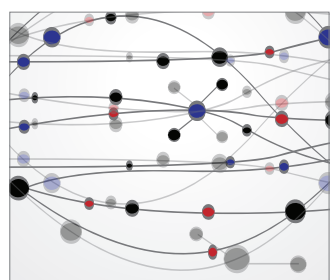

\section{The Scientific} World Journal
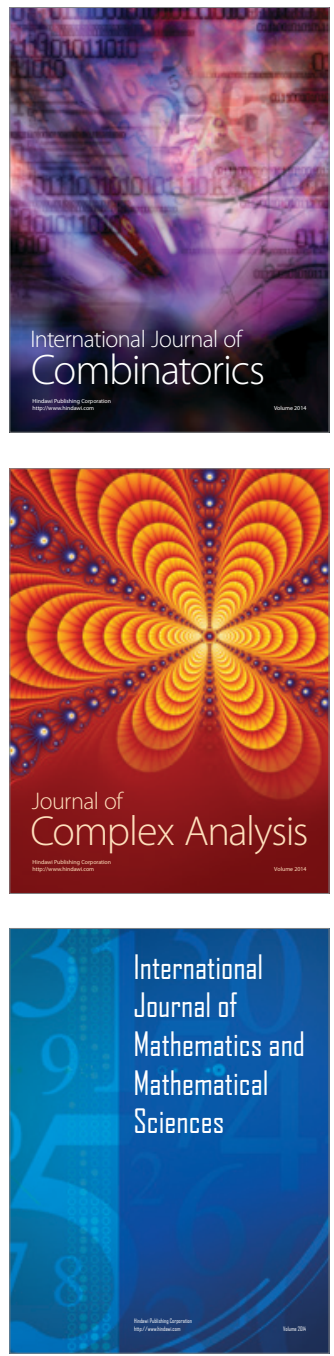
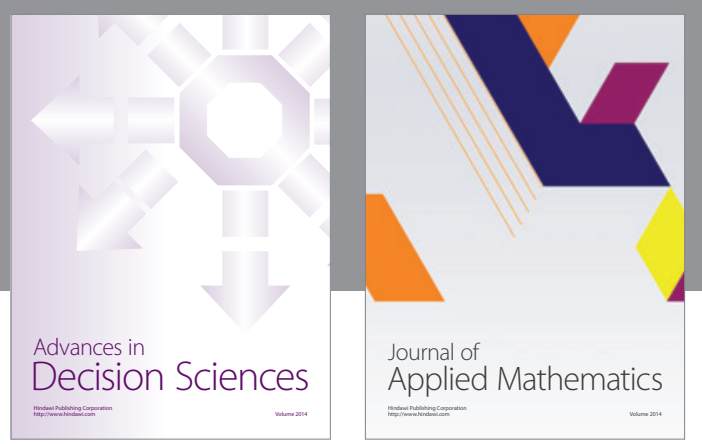

Algebra

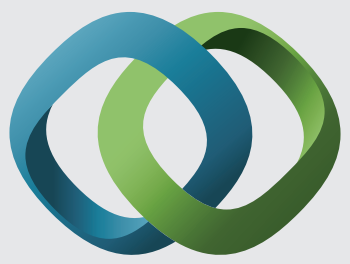

\section{Hindawi}

Submit your manuscripts at

http://www.hindawi.com
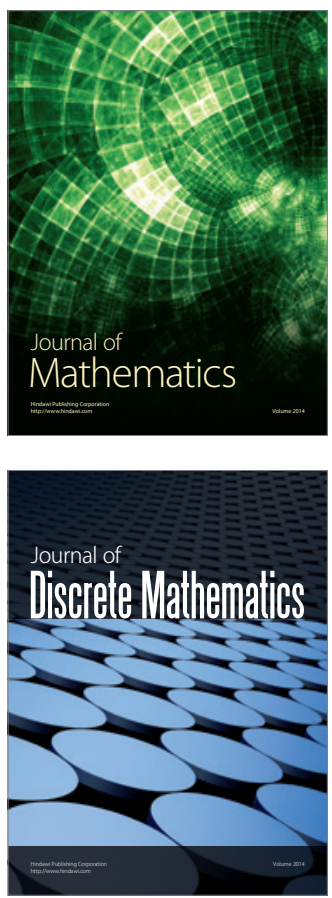

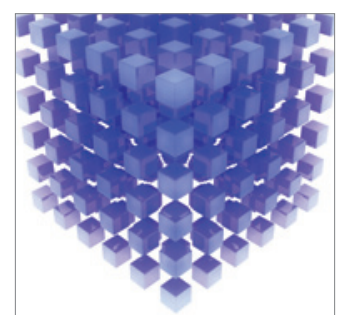

Mathematical Problems in Engineering
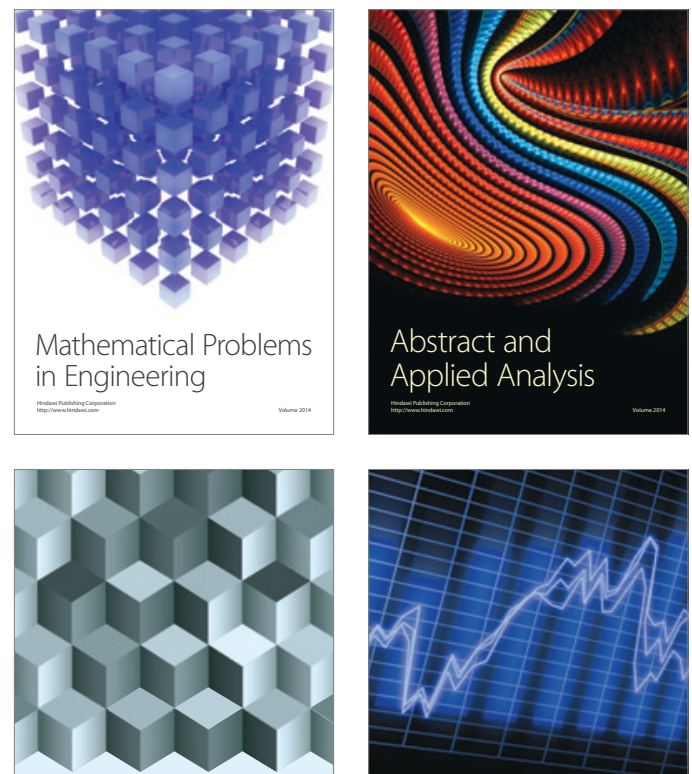

Journal of

Function Spaces

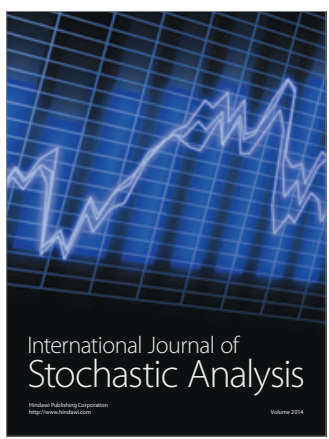

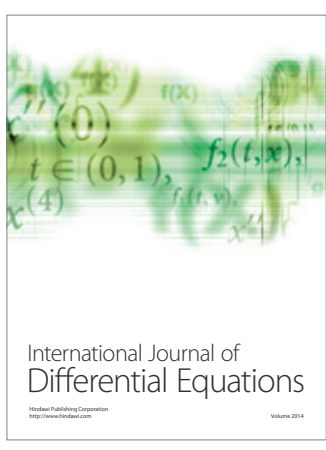
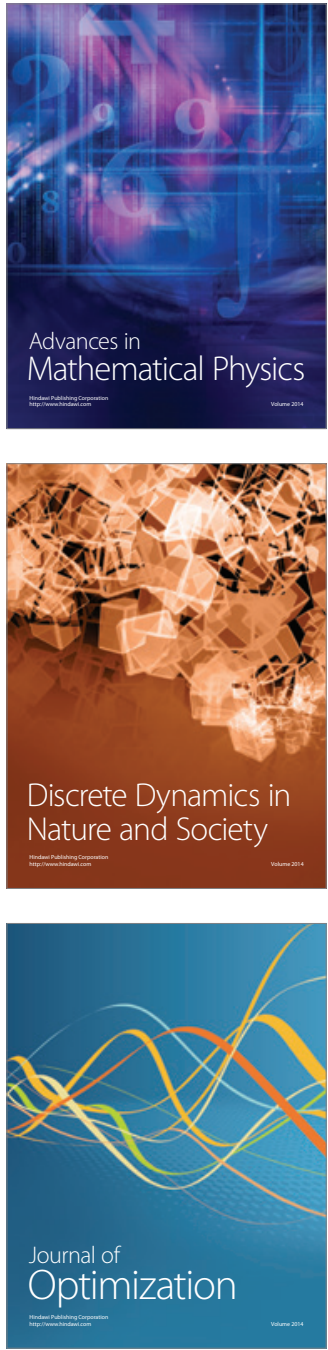